\title{
Spin Distributions and Dynamics in Domain Walls Guided by Soft Magnetic Nanowire Structures
}

\author{
Jusang Yang $^{1, *}$ and James L. Erskine ${ }^{1}$ \\ ${ }^{1}$ Department of Physics, The University of Texas at Austin, Austin, Texas 78712-1081, USA
}

\begin{abstract}
Numerical simulations are used to investigate static and dynamic properties of spin distributions within domain walls confined by rectangular cross section Permalloy nanowire conduits having widths up to $1000 \mathrm{~nm}$ and thickness up to $50 \mathrm{~nm}$. Phase boundaries and critical regions associated with domain-wall spin distributions of various topologies [transverse(or asymmetric transverse), vortex, double-vortex, triple-vortex and cross-tie] are accurately determined using high-performance computing resources. Mobility curves are calculated that characterize domain-wall propagation for an interesting region of the spin texture phase diagram: $20 \mathrm{~nm}$ thick nanowires with widths of $60-700 \mathrm{~nm}$ at axial drive fields extending to 150 Oe. The simulations (and corresponding experiments, which are discussed), reveal new propagating fixed configuration domain-wall topologies with enhanced velocity. Effects of temperature on the spin distributions and dynamics are explored, by conducting simulations that include separately varying temperaturedependent parameter (saturation magnetization and exchange constant) and simulating effects of temperature-dependent fluctuations using the Langevin dynamics feature of the simulation code. Related temperature-dependent experiments are discussed. The simulation studies demonstrate a close connection between static and (field-driven) dynamic spin configurations in nanowire-confined domain walls and demonstrate the importance of exploring model-system parameter space at high numerical precision.
\end{abstract}

Keywords: Domain-wall phase diagram; Domain-wall dynamics; Temperature-dependent dynamics

\footnotetext{
${ }^{*}$ Electronic address: jsyang@physics.utexas.edu
} 


\section{INTRODUCTION}

Magnetostatic analysis shows that electron spins in soft magnetic material fabricated into nanowires align to form head-to-head or tail-to-tail magnetic domains (regions of inplane magnetic saturation) separated by domain walls (DWs) having widths of a few hundreds of nanometers.[1] The properties of DWs confined and guided by nanowire conduits are technologically important: Magnetic nanowires can be used for storing and processing magnetically-coded information in logic and memory devices, $[2-4]$ and are being explored as the basis for lab-on-a-chip platforms for manipulating and functionalizing magnetic nanoparticles (trapped by DW stray fields) for biomedical applications[5-7]. Thin-film based magnetic nanowire structures have also become an important venue for probing fundamental properties of magnetic-field[8-11] and electric-current[12-17] driven DW propagation in confined geometry model 1-dimensional (1D) structures.

The static spin distribution in soft magnetic nanowires (Permalloy, for example) is governed by the competition between shape anisotropy and exchange energies.[1] However, the complexity of cross-sectional-area dependent anisotropy energies requires numerical approaches $[18,19]$ to determine the spin distributions within a DW for prescribed nanowire geometry. Prior numerical simulations have explored the static spin distributions within DWs confined by rectangular Permalloy nanowires. McMichael and Donahue[18] showed that the phase boundary between transverse wall (TW) and vortex wall (VW) structures is governed by the empirical formula $t w=\eta l_{e x}^{2}$ where $t$ is the wire thickness, $w$ is the wire width and $l_{e x}=\sqrt{A /\left(2 \pi M_{\mathrm{s}}^{2}\right)}$ is the magnetic exchange length. The dimensionless constant $\eta=64$ was obtained by fitting the numerically-determined phase boundary. The other physical parameters for Permalloy are the exchange stiffness, $A=1.3 \times 10^{-6} \mathrm{erg} / \mathrm{cm}$ and saturation magnetization, $M_{\mathrm{s}}=800 \mathrm{emu} / \mathrm{cm}^{3}$. Note that the different dimensionless constant (136)[18] is due to the different definition of the exchange length, $\sqrt{A /\left(\mu_{0} M_{\mathrm{s}}^{2}\right)}(\mathrm{SI})=\sqrt{A /\left(4 \pi M_{\mathrm{s}}^{2}\right)}(\operatorname{cgs})$.

A refinement of the phase diagram for a rectangular cross section Permalloy wire was reported by Nakatani, Thiaville and Miltat.[19] They showed that the asymmetric transverse wall (ATW) which has the same topology of a TW existed between the TW and VW phases along a region of the TW-VW phase boundary. Nakatani et al. also explored the role of TW spin distributions within a propagating DW in governing the wall mobility by calculating the effective DW width parameter, $\Delta$, and correlating it with the drive-field de- 
pendent DW velocity (mobility) function $\mu(H)$. The Walker low-field analytical solution[21] for a field-driven TW predicts a propagation velocity described by $v=\mu H$ with $\mu=\gamma \Delta / \alpha$, where $\gamma=$ gyromagnetic constant, $\Delta=$ wall width parameter, and $\alpha=$ spin damping parameter. This (linear mobility) solution is valid for $H<H_{\mathrm{W}}$; above $H_{\mathrm{W}}$, oscillatory DW displacements driven by precessional motion invalidates the stationary TW assumption of the simple analytical model.

While the Walker analytical solution is compatible with mobility measurements and numerical simulations for low drive fields, DW mobility measurements[17] manifest critical fields $H_{\mathrm{c}}$ (characterized by the breakdown of linear mobility behavior) far below the analytical prediction for $H_{\mathrm{w}}$. Measured values of $H_{\mathrm{c}}$ for Permalloy nanowires are $H_{\mathrm{c}}=5$ Oe, whereas the analytical model predicts $H_{\mathrm{W}}=\alpha H_{\mathrm{K}} / 2=2 \pi \alpha M_{\mathrm{s}} \approx 50$ Oe where $H_{\mathrm{K}}$ is the transverse anisotropy field, $\left(H_{\mathrm{K}}=2 K /\left(4 \pi M_{\mathrm{s}}\right), K=\right.$ transverse anisotropy energy). Clearly the analytical model fails[9] at values of $H_{\mathrm{c}} \ll H_{\mathrm{W}}$; i.e., at values of $H$ far below the precessional mode regime associated with the Walker field. Numerical simulations account for this failure: Vortex DW formation becomes energetically favorable at critical fields $H_{\mathrm{c}}$ below the Walker field. The transformation of the DW motion to (precessional) vortex dynamics above an experimentally-determined critical field underlies the importance of numerical simulation of DW propagation above the critical field. While analytical models of precessional velocity oscillations $[1,10]$ are capable of providing a qualitative account of measurements[8, 10] and simulations[10, 22] above the critical field, numerical simulations offer the only realistic prospects for quantitative understanding of DW dynamics that are dominated by topologies other than simple TW structures.

\section{NUMERICAL SIMULATION METHOD}

This paper describes a systematic numerical simulation study of static and magnetic-field driven dynamic spin distributions in rectangular cross section Permalloy nanowires. The micromagnetic simulations were carried out using a version of LLG Micromagnetic Simulator developed by M. R. Scheinfein[23] and adapted to The University of Texas TACC Lonestar Cluster[24]. The Cray-Dell cluster is a 5840 core 64-bit Linux-based system capable of 62 TFLOP peak performance. Systematic numerical studies of parameter-dependent effects carried out using the Lonestar Cluster can be executed at a rate of 100 to 1000 times 
TABLE I. Temperature-dependent parameters for the saturation magnetization $M_{\mathrm{s}}$, and the exchange constant $A$ that were used in the numerical simulations.

\begin{tabular}{ccc}
\hline \hline$T(\mathrm{~K})$ & $M_{\mathrm{s}}\left(\mathrm{emu} / \mathrm{cm}^{3}\right)^{\mathrm{a}}$ & $A\left(\times 10^{-6} \mathrm{erg} / \mathrm{cm}\right)^{\mathrm{b}}$ \\
\hline 100 & 898.38 & 1.22 \\
200 & 851.67 & 1.12 \\
$300^{\mathrm{c}}$ & 800.00 & 1.00 \\
400 & 741.73 & 0.88 \\
500 & 674.11 & 0.74 \\
\hline \hline
\end{tabular}

${ }^{\text {a }} M_{\mathrm{s}} \propto\left(T_{\mathrm{c}}-T\right)^{\beta}, T_{\mathrm{c}}=833 \mathrm{~K}, \beta=0.364$. Ref. $[25,26]$

b $A(T) \propto M_{\mathrm{s}}^{2-\epsilon}, \epsilon=0.255$. Ref. [27]

${ }^{c}$ Parameters used in Secs. III and IV.

faster and at greater (64 bit) precision than on a (singe node) desk-top computer. This significantly-enhanced performance has allowed exploration of spin distribution phase diagrams and mobility curves on a broader and finer parameter-space scale than previously accessible. The improved numerical simulation resolution has revealed new static and dynamic spin topologies and static $(H=0)$ phase-boundaries of the spin configurations that are described in this paper.

All of the numerical simulations for both static and dynamic spin distributions were carried out using materials parameters appropriate for Permalloy $\left(\mathrm{Ni}_{80} \mathrm{Fe}_{20}\right)$ listed in Table I, and Gilbert damping constant $\alpha=0.01$ ( or $\alpha=2$ for static spin distributions). Simulations that explored the static phase boundaries covered rectangular dimensions that extended to $50 \mathrm{~nm}$ thickness and $1000 \mathrm{~nm}$ width. Domain-wall dynamics were studied in rectangular cross section wires of $20 \mathrm{~nm}$ thickness and selected widths ranging from $60 \mathrm{~nm}$ to $700 \mathrm{~nm}$ (which span an interesting region of the static phase diagram) for applied fields extending to 150 Oe and over time periods required to fully characterize the oscillatory DW displacements. The unit cell size and numerical integration time step in the simulations were $4 \times 4 \times 20 \mathrm{~nm}^{3}$ and $0.3 \mathrm{ps}$, respectively. To reduce the simulation system size, moving boundary conditions were adopted while keeping the DWs far away from the ends of nanowires. In the regions of viscous, oscillatory and vortex-antivortex resonance modes, the ratio of the length and the width of the simulation system size was 7.5 (i.e., for $400 \mathrm{~nm}$ width, the simulation system 


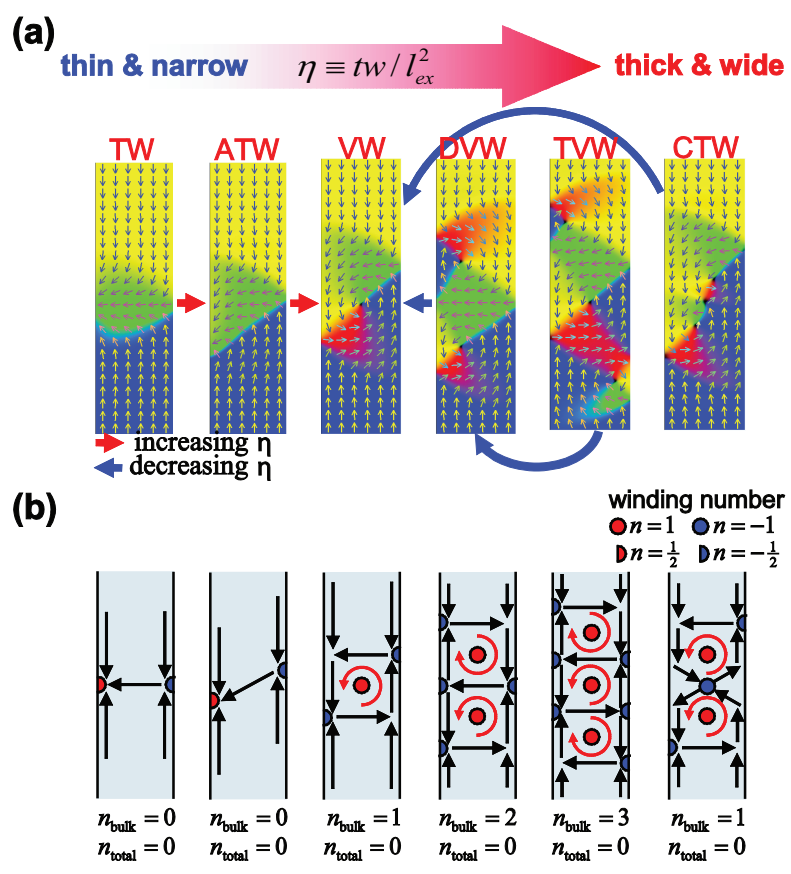

FIG. 1. (color online) Stable domain-wall spin distributions in a rectangular cross-section Permalloy nanowire as a function of cross-section parameter. (a) Simulations of transverse wall (TW), asymmetric transverse wall (ATW), vortex wall (VW), double-vortex wall (DVW), triple-vortex wall (TVW), cross-tie wall (CTW), (b) Schematic representations of domain-wall types showing topological winding numbers.

length was chosen to be $l=3 \mu \mathrm{m}$ ). For the stretching mode (described later), however, the ratio was 20 (i.e, for the $400 \mathrm{~nm}$ width, the simulation system length $l=8 \mu \mathrm{m}$ ), because the stretch mode DW tends to be elongated. The average DW velocity for an external field was obtained after the total simulation time of $\sim 100 \mathrm{~ns}$.

\section{STATIC SPIN DISTRIBUTIONS AND PHASE BOUNDARIES}

The stable DW types illustrated in Fig. 1(a) were found by relaxing a random spin configuration within the simulation volume subject to the boundary conditions that define the nanowire geometry. Nanowires with small cross-sectional area (low values of the system size parameter $\left.\eta \equiv t w / l_{\mathrm{ex}}^{2}\right)$ support TW or VW structures. Thicker and wider nanowires support various types of DWs such as double-vortex wall (DVW), triple-vortex wall (TVW) and cross-tie wall (CTW).

Figure 1(b) shows simplified schematic diagrams of the six distinct compact DW types 

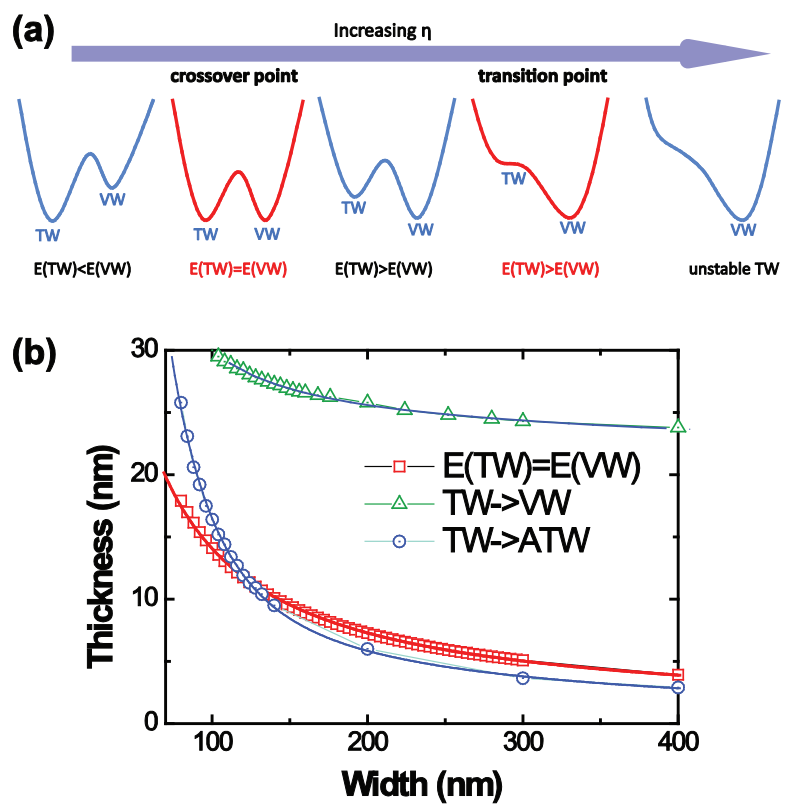

FIG. 2. (color online) (a) Schematic representations of the energy landscape for the crossover point and transition point of the transformation from a transverse wall to a vortex wall as a function of the cross-sectional area parameter. (b)Phase diagram for TWs and VWs.

that were identified by numerical simulations and illustrates topological properties of these six spin configurations. The TW (or ATW) has two fractional topological edge defects (winding number $n=-\frac{1}{2}$ and $+\frac{1}{2}$ ), while the VW has two $-\frac{1}{2}$ edge defects and one +1 bulk defect. The DVW has three $n=-\frac{1}{2}$ edge defects and two +1 bulk defects, while the TVW is composed of six $n=-\frac{1}{2}$ edge defects and three +1 bulk defects. The CTW is made of two $-\frac{1}{2}$ edge defects, two +1 bulk defects, and one -1 bulk defect. The sum of the winding numbers of edge defects is thus the negative sum of the winding numbers of bulk defects, making the total sum of winding numbers to be zero.

Crossover and transition boundaries of the DW phase diagram are determined by the energy diagram schematics in Figure 2(a). The phase diagram boundaries shown in Fig. 2(b) were determined by starting with one of the stable configurations (initial spin distribution) shown in Fig. 1(a). The initial spin distribution was obtained by starting with a random spin configuration for a prescribed set of magnetic conduit cross-sectional dimensions. The thickness $t$ was then incrementally increased (when starting from a TW) or incrementally decreased (when starting from any of the other stable wall configurations) and the DW spin configuration was allowed to relax to the lower energy configurations. Thickness incre- 
ments were reduced to $0.05 \mathrm{~nm}$ near transition regions and phase boundaries to improve the accuracy of boundary parameters.

Figure 2(a) shows schematic representations of the global DW energy as a function of the cross sectional area parameter $\eta \equiv t w / l_{\mathrm{ex}}^{2}$ near what we define as a crossover point and a transition point between a transverse and a vortex wall spin distributions. A crossover point corresponds to one point on a line of the width vs thickness diagram where the system energies of two DW spin configurations are equal. Both phases can coexist in regions near a crossover point. A transition point corresponds to a point on a line on the width vs thickness diagram where a saddle point occurs for one of the DW spin configurations. This line defines a phase boundary.

The entire phase diagram that characterizes the crossover and phase boundaries for all six DW configurations for rectangular cross section Permalloy nanowires is fairly complicated, therefore, it is useful for the purpose of clarity, to present the phase diagram for subsets of the allowed phases separately.

Figure 2(b) displays the phase diagram for formation of TW, ATW and VW DW spin configurations. It shows one crossover boundary $[E(\mathrm{TW})=E(\mathrm{VW})]$ and two transition boundaries $(\mathrm{TW} \rightarrow \mathrm{ATW}$ and $\mathrm{TW} \rightarrow \mathrm{VW})$. The crossover boundary $[E(\mathrm{TW})=E(\mathrm{VW})$ in Fig. 2(b)] was previously reported by McMichael and Donahue[18] and our simulation accurately reproduces their result. Nakatani et al.[19] reported that the ATW area falls inside the TW area. However, our phase diagram shows that the ATW extends into the area where the VW is a global minimum configuration [i.e., the line associated with $\mathrm{TW} \rightarrow \mathrm{ATW}$ in Fig. 2(b)]. Consistent simulation results have recently been reported by S. Jamet et al.[20] The $\mathrm{TW} \rightarrow \mathrm{VW}$ transition boundary is experimentally more important than the crossover boundary because below the $\mathrm{TW} \rightarrow \mathrm{VW}$ transition boundary, TW and VW can coexist and can be transformed into each other by thermal activation or applied external magnetic fields, but above this boundary only VW spin configurations can form.

We explored more detailed phase boundaries [the remaining 3 spin configurations in 1(a)] by including DVW, TVW, and CTW as initial spin configurations. Figure 3 displays the domain-wall energy as a function of nanowire thickness for all possible compact spin configurations that can form in a nanowire of width $300 \mathrm{~nm}$. This energy diagram corresponds to a vertical line (not shown) on Fig. 2(a) at $w=300 \mathrm{~nm}$. Starting at $60 \mathrm{~nm}$ thickness where TVW, DVW, CTW and VW are all possible, and choosing a TVW as a starting con- 


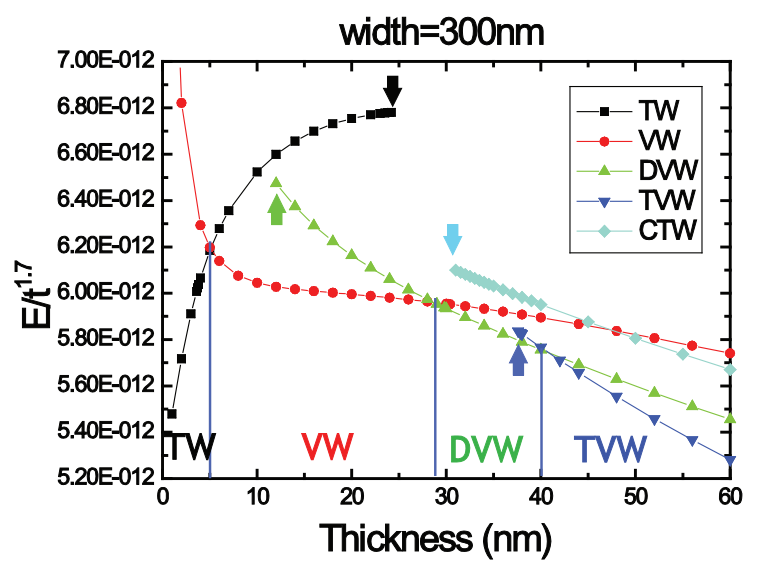

FIG. 3. (color online) Plot of the domain-wall energy as a function of nanowire thickness for a fixed-width nanowire. The arrows indicate transition boundary points. The crossover boundaries are determined by domain-wall energy crossing points.

figuration, the TVW transforms into a DVW at about $38 \mathrm{~nm}$ as the thickness is decreased. Starting with a CTW, the CTW transforms to a VW at about $32 \mathrm{~nm}$ thickness, while the DVW transforms to a VW at about $12 \mathrm{~nm}$ as the thickness is decreased. The VW, once stabilized at $60 \mathrm{~nm}$ thickness, is stable (global minimum or local minimum states) over the entire thickness region studied. The DW spin configuration transition points are indicated in Fig. 3 by arrows. Note that for the convenience of visualization the domain-wall energies were divided by $t^{1.7}$.

The crossover boundaries were determined from the energy crossing points. The global minimum spin configuration becomes TW, VW, DVW, and TVW in turn as the crosssectional area of a nanowire is increased. However the CTW exhibits only a local minimum state.

Figure 4 shows the phase diagram of domain-wall types with the thickness parameter extended to $50 \mathrm{~nm}$ and width parameter extended to $1000 \mathrm{~nm}$. Note that some phase boundaries already shown in Fig. 2(b) are not included to simplify the figure. There are three main regions: In the region Ia (TW=global minimum, VW=local minimum), Region Ib (TW= global minimum, VW/DVW=local minimum); Region IIa (VW= global minimum, TW=local minimum), Region IIb (VW=global minimum, TW/VW/DVW=local minimum), Region IIc (VW=global minimum, VW/DVW=local minimum); Region IIIa (DVW=global minimum, $\mathrm{TW} / \mathrm{VW}=$ local minimum, $\mathrm{VW}=$ local minimum). The $\mathrm{CTW}$ is 


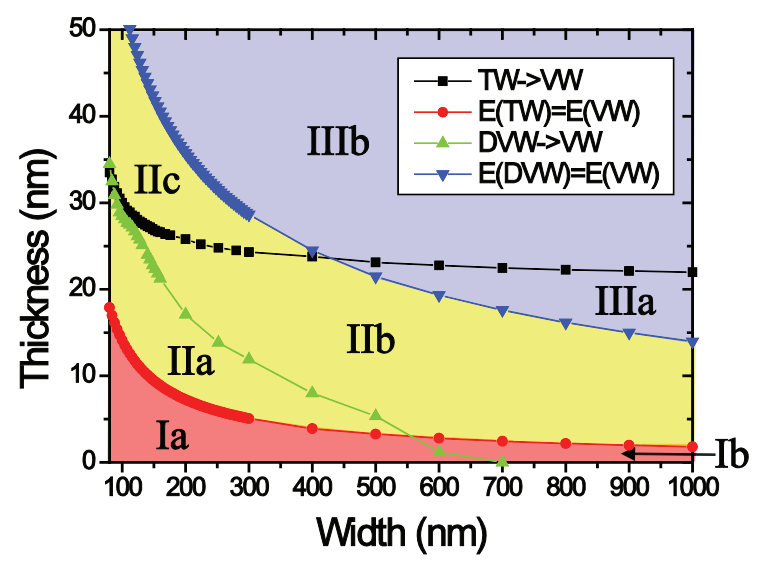

FIG. 4. (color online) Domain-wall spin distribution phase diagram showing crossover boundaries and transition boundaries for several DW types in rectangular Permalloy nanowires.

not a global minimum configuration in the whole region we studied. In the case of a TVW, it has a global minimum state at just above the $E(\mathrm{DVW})=E(\mathrm{VW})$ crossover boundary (not shown here).

Note that the transition boundary of DVW $\rightarrow \mathrm{VW}$ is below $t=20 \mathrm{~nm}$ for the widths of $w>200 \mathrm{~nm}$. Therefore, we can expect more complex dynamic behavior for the large cross-sectional nanowires. It is not easy to experimentally control the injected domain-wall types due to the coexistence of several (local) minimum states for various cross section parameters. In addition, a pre-determined domain-wall type can be transformed into other types by external parameters such as bias fields, driving fields, spin torque and thermal activation.

\section{DOMAIN WALL DYNAMICS}

Figure 5 displays numerically simulated mobility curves $v(H)$ for $20 \mathrm{~nm}$-thick rectangular cross section Permalloy nanowires ranging in width from $60 \mathrm{~nm}$ to $700 \mathrm{~nm}$. Note from the two static phase diagrams (Figs. 2 and 4) that a line at the chosen thickness of $20 \mathrm{~nm}$ approaches or crosses several phase boundaries. Vortex domain walls were used as the initial state in the dynamic response simulations, because the VW configuration is a global minimum or a local minimum state over the entire region studied.

Several general trends in the mobility curves in Fig. 5 can be observed: 1) All of the mobility curves exhibit a region of linear mobility that extends from low drive fields up to 


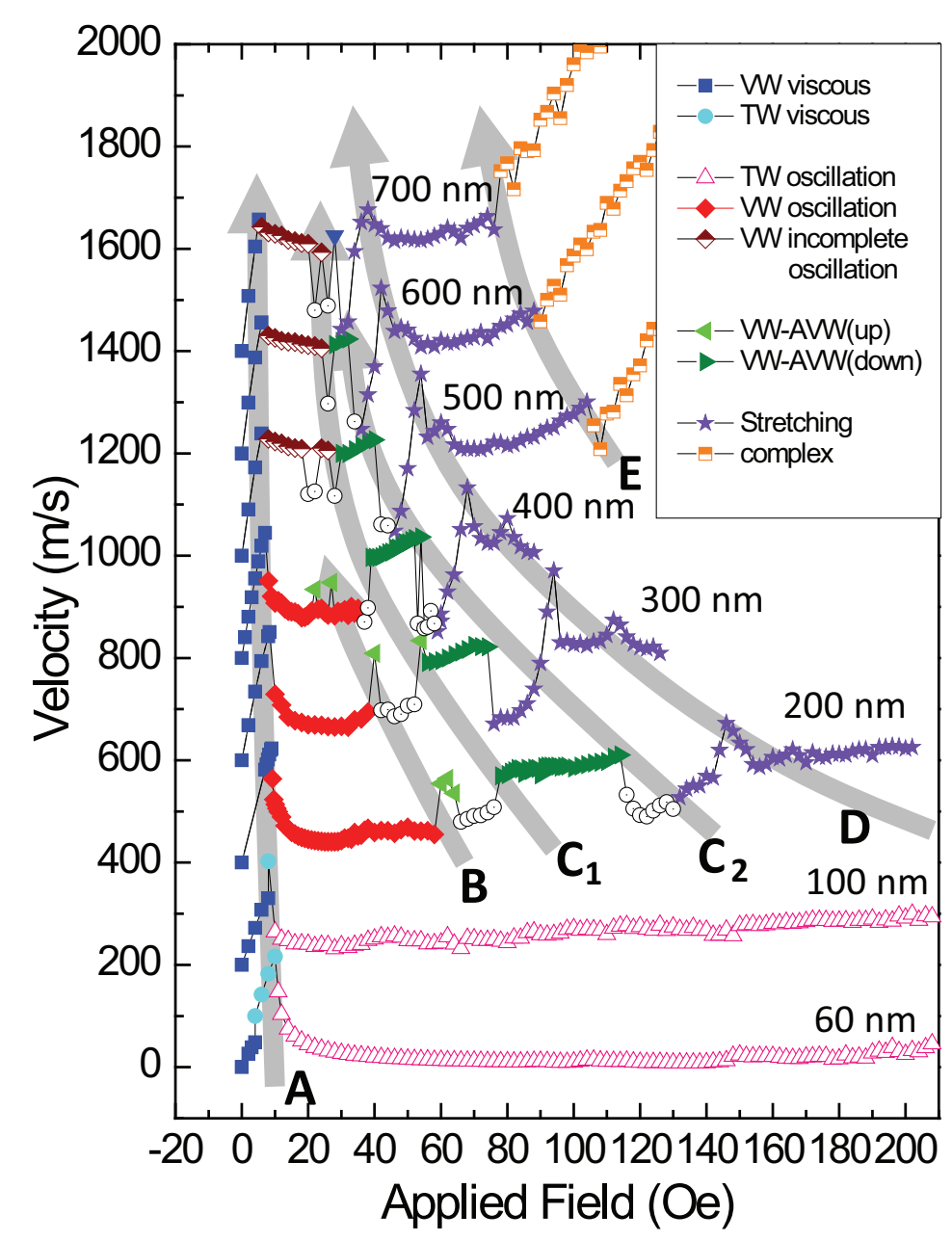

FIG. 5. (color online) Domain-wall mobility curves for 20 nm-thick rectangular Permalloy nanowires as a function of nanowire width. The region of the phase diagram (Fig. 4) spanned by the nanowire cross-section parameters includes the VW-DVW boundary. Several distinct regions of enhanced DW velocity are identified by different symbols and colors. Shaded arrows, identified by letters, track the nanowire width dependence of the drive-field regions of enhanced DW velocity.

the critical field $H_{\mathrm{c}}=10$ Oe. The weak dependence of $H_{\mathrm{c}}$ on nanowire width is shown in Fig. 5 by a shaded arrow labeled $A$, and is plotted in Fig. 6 along with the width dependence of other structures identified by shaded arrows labeled $B, C_{1}, C_{2}, D$, and $E$. 2) The region of negative differential mobility for $H>H_{\mathrm{c}}$ becomes less pronounced and narrower as a function of $H$ as nanowire width is increased, and the upper limit is pushed to lower values 


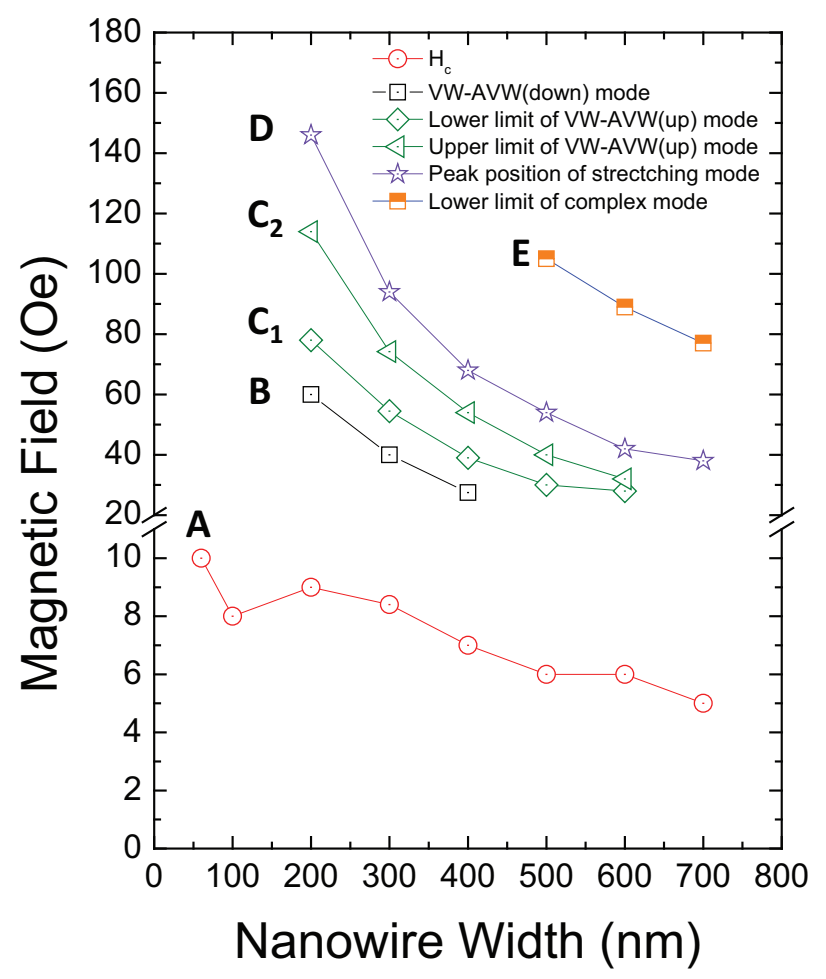

FIG. 6. (color online) Plot of the enhanced velocity dynamic mode drive-field boundaries and critical fields for $20 \mathrm{~nm}$-thick rectangular Permalloy nanowires as a function of wall width.

of drive field for nanowire widths above $100 \mathrm{~nm}$ as new modes with higher propagation velocity emerge. 3) Nanowires having widths above $100 \mathrm{~nm}$ support several new modes that form over regions of higher $H$. These new modes exhibit higher propagation velocity (the new modes are denoted by $B, C_{1}, C_{2}, D$, and $E$ in the figure, and shaded arrows illustrate the width-dependent drive field shifts).

The critical field, $H_{\mathrm{c}}$ and the onset of new modes for nanowire widths above $100 \mathrm{~nm}$ shift to lower drive-fields as the nanowire width is increased (as shown in Fig. 6). The DW spin configuration in the $60 \mathrm{~nm}$ wide nanowire is a TW structure near $H=H_{\mathrm{c}}$, however stable VW propagation (square symbols at near zero drive fields) is observed in the $60 \mathrm{~nm}$ wide nanowire below 4 Oe. These propagating mode structures are consistent with the static phase diagram. All $20 \mathrm{~nm}$-thick nanowires having widths equal to or greater than $100 \mathrm{~nm}$ exhibit uniform (non-oscillatory) VW propagation for longitudinal fields below $H_{\mathrm{c}}(\leq 10 \mathrm{Oe})$. The simulations for the $100 \mathrm{~nm}$-wide case at $H=9$ Oe yield a stable TW structure near $H_{\mathrm{c}}$ 
(single round symbol at $H_{\mathrm{c}}$ ). The selected $100 \mathrm{~nm}$ width for the mobility curves displayed in Fig. 5 places the $20 \mathrm{~nm}$-thick nanowire near the TW-VW phase boundary (refer to Fig. 4). Based on the observation (refer to Fig. 5) that this specific nanowire geometry supports VW structures below $H=9$ Oe, it is possible that the applied longitudinal field is responsible for stabilizing the TW structure observed near the critical field where TW oscillations begin to occur in the $60 \mathrm{~nm}$ and $100 \mathrm{~nm}$ wide structures. Note that for $H>H_{\mathrm{c}}$ the oscillating TW mode remains stable throughout the entire drive field range studied (to 400 Oe, not shown in Fig. 5) only in the $60 \mathrm{~nm}$ and $100 \mathrm{~nm}$ wide structures. All wider widths exhibit DW dynamic mode transformations as a function of drive field $H$.

Domain-wall motion just above the critical field is characterized by negative differential mobility for all nanowire widths studied (up to $700 \mathrm{~nm}$ ). The reduced mobility for nanowire widths $w<200 \mathrm{~nm}$ results from oscillatory motion of TWs. The reduced mobility for $w>200 \mathrm{~nm}$ is caused by the oscillatory motion of vortex cores that sweep back and forth between the nanowire boundaries, changing core polarity at each wall reflection (discussed in more detail in relation to Fig. 7). The average forward motion of the core is slowed by the oscillatory displacement along the wire as $H$ increases which accounts for the negative differential mobility. This region of complex oscillatory motion has been explored experimentally[8, 10] and theoretically by analytical models[10] and by prior numerical simulations[10, 22, 28].

Our high-resolution simulations show that $20 \mathrm{~nm}$-thick nanowires having widths of $200 \mathrm{~nm}$ and greater, exhibit regions of drive-field strength over which a stable VW-AVW pair forms (green triangular symbols in Fig. 5). This stable spin configuration propagates at a significantly higher velocity than that associated with the oscillatory motion of single VW propagation. In addition, a second distinct high mobility non-stationary spin distribution (stretching mode) is observed at higher applied fields as the nanowire width is increased (blue star symbols in Fig. 5 with peak field designated by $D$ ). Figure 6 shows a graph of the systematic shift of $H_{c}$ and the onset shifts of high mobility structures to lower drive field as the nanowire width is increased.

Figure 7 isolates the simulated mobility curve for the $300 \mathrm{~nm}$-wide and $20 \mathrm{~nm}$-thick nanowire structure and describes, in greater detail, the DW motion by illustrating the spatial average of displacement and velocity records associated with selected drive-field regions designated by Roman numerals and symbols having different color. Region i $\left(0<H<H_{\mathrm{c}}\right)$ is 

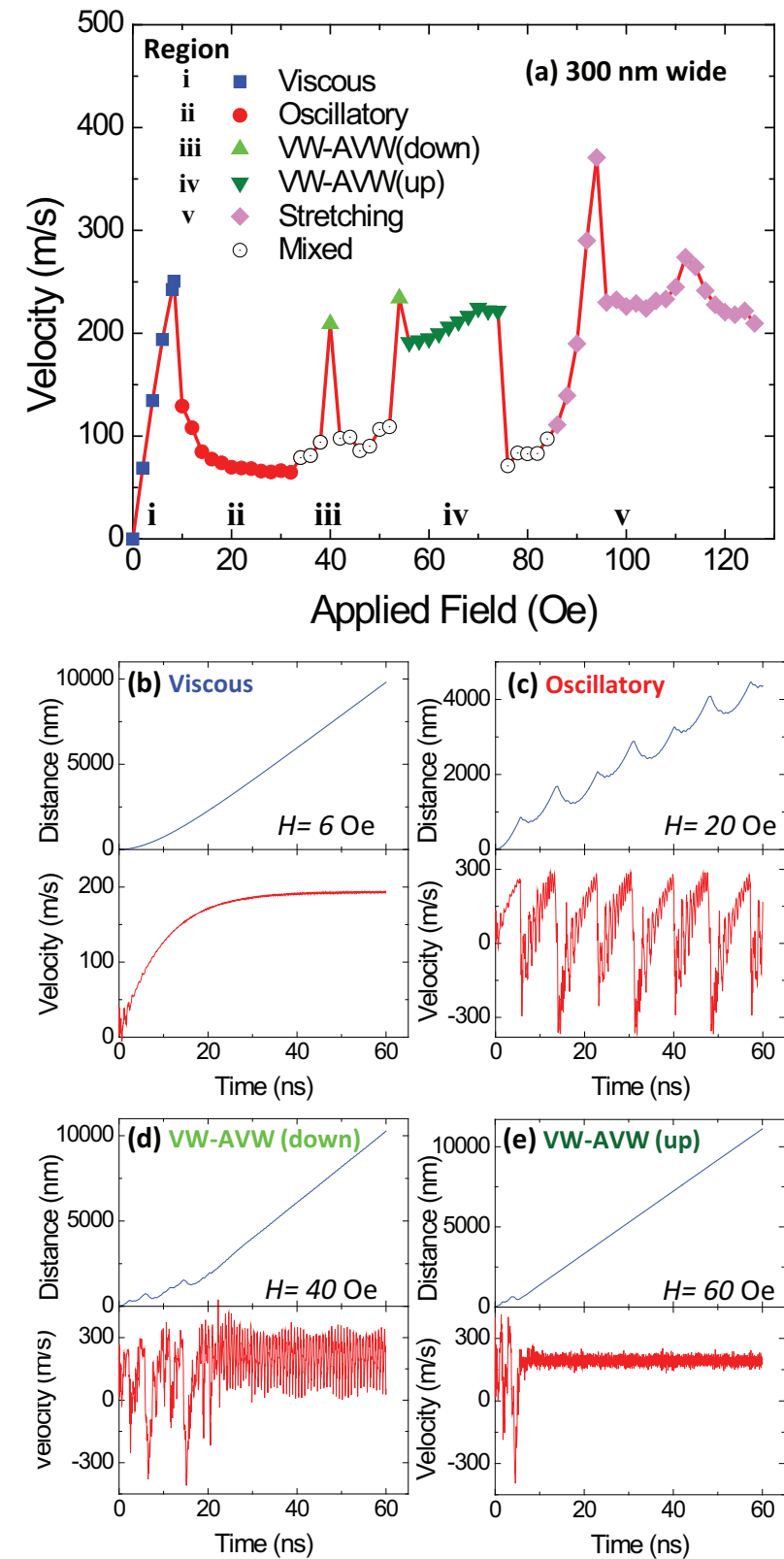

FIG. 7. (color online) Plots of the mobility curve, displacement records, and velocities for four distinct DW modes for a $300 \mathrm{~nm}$ wide and $20 \mathrm{~nm}$ thick nanowire. (a) Mobility curve showing five regions of specific mode propagation. Note that the specific parameters for this case correspond to Region iib of the static phase diagram where (single) vortex domain walls are predicted to exist. (b-e) Displacement and velocity records for specific domain wall types: b) stationary VW at $H=6$ Oe; c) oscillatory VW at $H=20 \mathrm{Oe}$; d) stationary VW-AVW(down) at $H=40$ Oe; e) stationary VW-AVW(up) at $H=60$ Oe. 
characterized by an essentially linear mobility behavior with mobility equal to approximately $30 \mathrm{~m} / \mathrm{sOe}$. Corresponding displacement and velocity plots [Fig. 7(b) for $H=6$ Oe] are labeled Viscous Region i. In this region, the (vortex spin configuration) DW accelerates to a terminal velocity of $194 \mathrm{~m} / \mathrm{s}$ in a time constant of $9.4 \mathrm{~ns}$ with a velocity curve accurately described by an exponential function. In Region ii (above the critical field, $H_{\mathrm{c}} \approx 8.4 \mathrm{Oe}$ ) the velocity oscillates between $\pm 300 \mathrm{~m} / \mathrm{s}$ with high regularity in the velocity record observed for every second cycle (the repeat period is about $17 \mathrm{~ns}$ for $H=20 \mathrm{Oe}$ ). The displacement record also exhibits the 17 ns period with an average slope of the distance vs. velocity record corresponding to $v \approx 70 \mathrm{~m} / \mathrm{s}$. This oscillatory mode persists from $H_{\mathrm{c}}$ to $H \approx 40$ Oe where two high-velocity modes forms over two drive-field ranges (a narrow Regions iii centered around 40 Oe, and a broader Region iv that extends from 50 to 75 Oe). Regions iii and iv are characterized by coupled vortex/antivortex pairs that propagate as quasi-stationary spin configurations at high velocity. The two modes have slightly different structure and time records [described in Figs. 7(d) and 7(e), and in Fig. 8(b)]. The lower field highmobility structure [Region iii, VW-AVW(down)] is less stable and exhibits larger-amplitude high-frequency velocity oscillations, but with a high average velocity of approximately $v=$ $209 \mathrm{~m} / \mathrm{s}$. The higher field structure (Region iv) becomes stable with small-amplitude highfrequency oscillations around $195 \mathrm{~m} / \mathrm{s}$ after $10 \mathrm{~ns}$ (the lower field mode requires over $20 \mathrm{~ns}$ to stabilize), and reaches a maximum average velocity of about $223 \mathrm{~m} / \mathrm{s}$ at $H=75$ Oe. Figure 8 shows a spatial description of the time history of a single vortex core [Fig. 8(a)] corresponding to Region ii where oscillatory propagation occurs, and a spatial time history [Fig. 8(b)] of the two types of coupled vortex/antivortex core structures, including spin texture diagrams (Regions iii and iv) where oscillations are suppressed and the DW propagates at high velocity.

Applied fields above 80 Oe produce a stretching mode that propagates at high velocity around $H=94$ Oe. The stretching mode is not one of the compact DW configurations and is a special type of mode that is affected dramatically by an applied magnetic field perpendicular to the nanowire axis and the thickness axis. This mode has been described in detail in prior publications[28], and will be briefly discussed in Section VI that outlines experimental evidence for the various modes described above. 

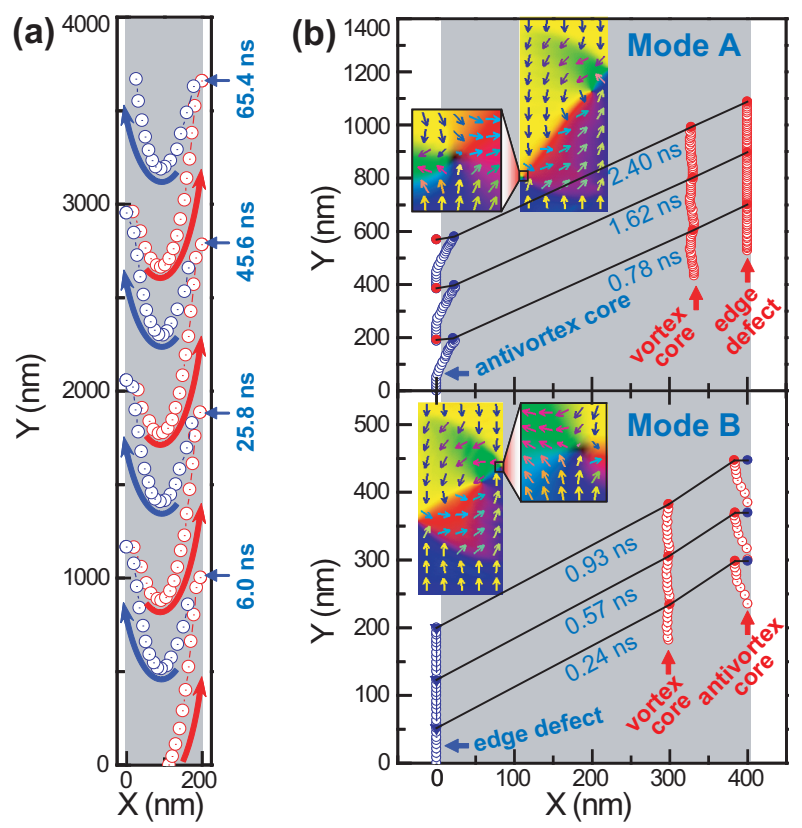

FIG. 8. (color online) Numerical simulations of DW spin distributions and trajectories. (a) Trajectory of a vortex core (in a $200 \mathrm{~nm}$ wide wire, $H=25$ Oe) showing core polarity reversal at wire edges accompanied by velocity oscillation (Region ii). (b) Trajectories of topological defects (edge defect, vortex core, and antivortex core) in a $400 \mathrm{~nm}$ wide wire, corresponding to coupled vortex-antivortex mode (Region iii). Insets show spin distributions with magnified antivortices. Antivortices are periodically generated at the left edge in Mode A $\left(H=27\right.$ Oe, $\left.H_{\text {bias }}=0\right)$; at the right edge in Mode $\mathrm{B}\left(H=47 \mathrm{Oe}, H_{\text {bias }}=0\right)$. Colors of symbols in (a) and (b) represent polarities of topological defects: red (+), blue (-).

\section{EFFECTS OF TEMPERATURE}

All of the results from numerical simulations presented in Figs. 1-8 were carried out using room temperature values of the saturation magnetization and the exchange constant, and neglecting local temperature-dependent fluctuations. Most experimental studies of domainwall dynamics in Permalloy nanowires have been carried out at room temperature. However, experiments that study spin-torque effects on DWs guided by nanowire conduits usually involve higher temperatures due to Joule heating resulting from the high current density $\left(\sim 10^{12} \mathrm{~A} / \mathrm{m}^{2}\right)$ required to observe spin-torque effects. Therefore, knowledge of temperature effects on DW structure and propagation could be important in some cases.

A series of numerical simulations were carried out for $200 \mathrm{~nm}$ wide $20 \mathrm{~nm}$ thick Permalloy 


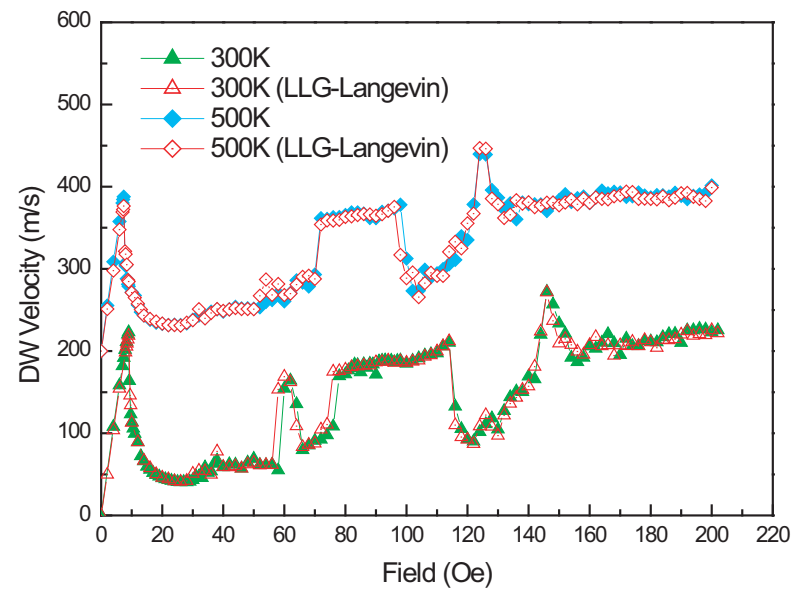

FIG. 9. (color online) Numerical simulations of the temperature dependence of the mobility curves for a $300 \mathrm{~nm}$ wide $20 \mathrm{~nm}$ thick Permalloy nanowire. The red-open symbols represent LLG simulations including thermal fluctuation term.

nanowires in which the values of $M_{\mathrm{S}}$ and $A$ corresponding to temperatures between $T=100$ and $500 \mathrm{~K}$ were changed independently and together to compare selected simulated features of static and dynamic domain-wall behavior as a function of temperature. To assess any additional contribution from the thermal fluctuations, numerical simulations including the stochastic term were also carried out for $T=300$ and $500 \mathrm{~K}$ and are shown in Fig. 9. The choice of cross sectional dimensions for the simulations was based on results of Fig. 5 that suggests specific cross section parameters where interesting dynamical effects lead to regions of enhanced velocity due to mode changes involving spin distributions of nearly equal magnetic system energy. Table I lists the temperature-dependent values of magnetization and exchange constants used in the calculations.

Complex temperature dependent behavior was observed in the simulation results, but a few general trends can be noted: 1) The effect of the thermal noise term can be ignored in the macroscopic domain wall dynamics. 2) Simulations of drive-field dependent local properties (precession frequencies and relaxation times) are consistent with analytical models. 3) Mobility curves exhibit systematic temperature dependent shifts that are similar to static magnetic bias-field shifts reported in prior publications[28] and which are consistent with shifts of measured mobility curves described later.

Figure 9 shows that the DW velocity curves from the stochastic and non-stochastic simulations are almost identical and there are only a slight deviation near the dynamic tran- 


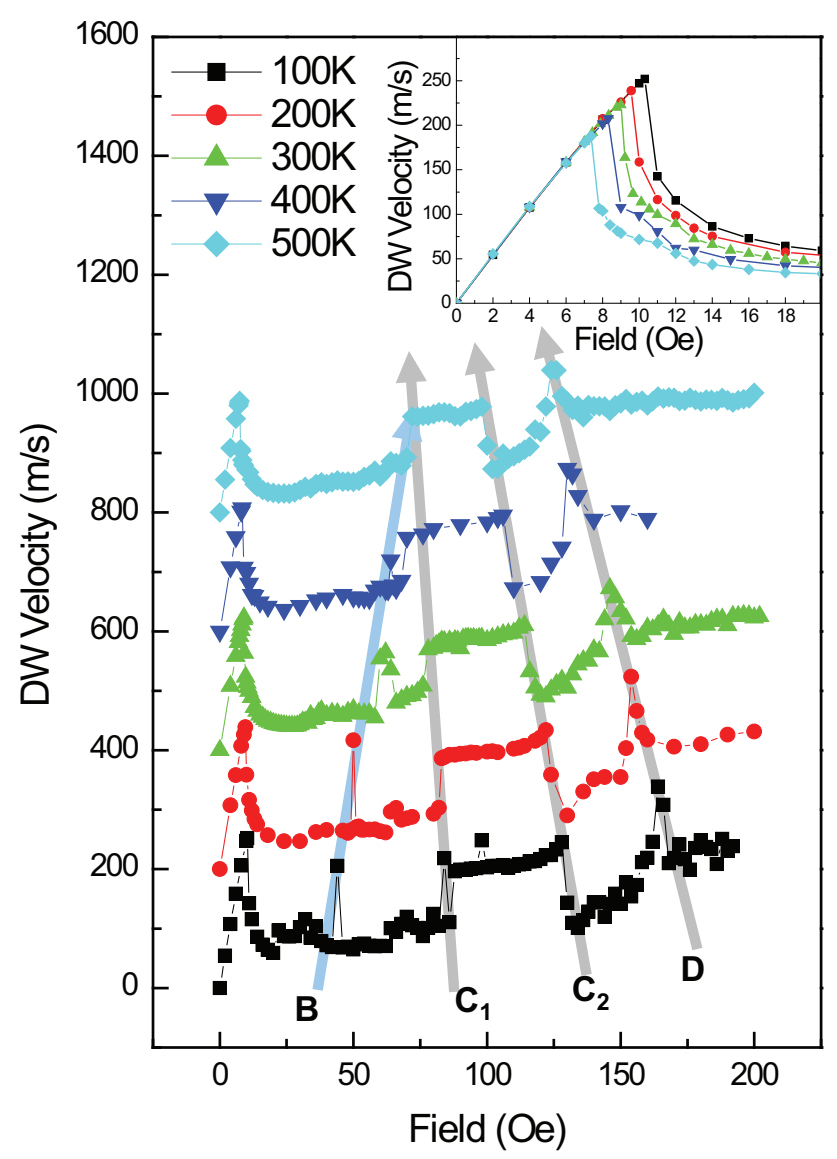

FIG. 10. (color online) Numerical simulations of the temperature dependence of the mobility curves for a $300 \mathrm{~nm}$ wide $20 \mathrm{~nm}$ thick Permalloy nanowire. Shaded arrows track the drive-field temperature dependence of the enhanced velocity regions (same notation as in Fig. 7). Inset shows the temperature-dependent shift for the critical field.

sitional regions. The major change in the DW velocity curve comes from the temperature dependence of of $M_{\mathrm{S}}$ and $A$.

Figure 10 presents temperature-dependent mobility curves corresponding to one of the nanowire structure mobility curves in Fig. 5 including temperature values above and below room temperature. The primary effect of temperature change is to shift the drive field range where stable compact DW modes exist. The shifts are indicated by shaded arrows that track three of the stable modes in the mobility curves as the temperature changes. An inset shows an expanded graph at low drive fields where the shift in critical field occurs. As the temper- 


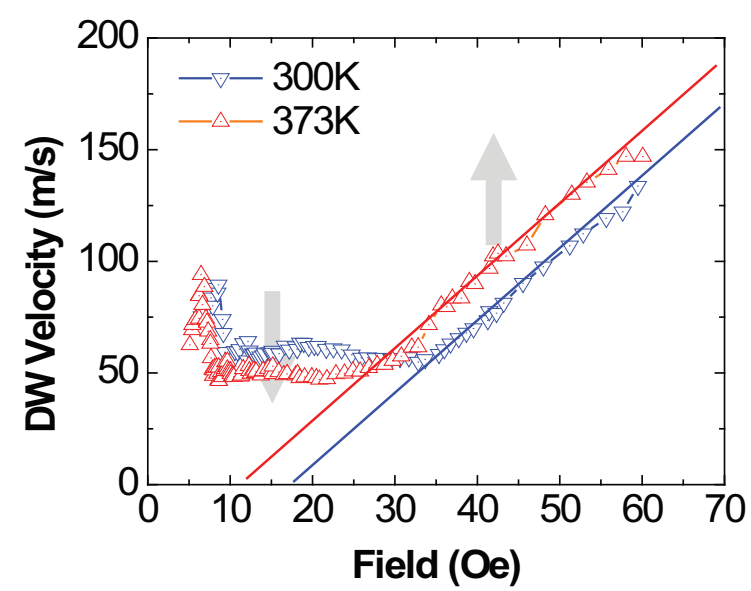

FIG. 11. (color online) Experimentally determined mobility curves at two temperatures for a $600 \mathrm{~nm}$ wide $20 \mathrm{~nm}$ thick Permalloy nanowire.

ature increases, the stretch mode, the VW-AVW(up) mode and the transition to negative differential mobility (critical field) shift to lower drive fields, but the VW-AVW(down) mode shifts to higher drive fields and eventually merges with the VW-AVW(up) mode.

It is not surprising that temperature changes in the range covered $(100-500 \mathrm{~K})$ do not destroy the DW mode structures. The shape anisotropy energy in soft magnetic materials, including Permalloy, dominates other anisotropy energies, and accounts for the observed in-plane magnetization in Permalloy thin films and the axial magnetization in Permalloy nanowire structures. The shape anisotropy energy in Permalloy is also large compared to thermal energies at and near room temperature, therefore static and dynamic spin distributions in domain walls are not expected to depart significantly from zero temperature properties in Permalloy nanowire structures as the temperature is changed. However, some of the domain-wall behavior observed in the simulations (such as mode hopping between spin configurations having nearly equal energies) and shifts of the field and temperature dependent onsets of various spin configurations is not unexpected. We again note that very similar drive field dependent shifts in mobility curve structures have been reported in studies in which the variable parameter is a static bias magnetic field perpendicular to the nanowire.[28]

Figure 11 displays experimentally measured mobility curves for a $600 \mathrm{~nm}$ wide $20 \mathrm{~nm}$ thick Permalloy nanowire at room temperature $(300 \mathrm{~K})$ and at $373 \mathrm{~K}$. The DW mobility 
curves in Fig. 11 and other mobility curves[8, 17] provide evidence of the enhanced velocity coupled vortex modes characterized by simulations and described by Figs. 5, 6, 8 and 10 . Comparing the $300 \mathrm{~K}$ and $373 \mathrm{~K}$ mobility curves in Fig. 11 it is apparent that the structure in the $300 \mathrm{~K}$ mobility curve that extends from 10 to 30 Oe drive field is suppressed at higher temperature and that linearly increasing mobility above 35 Oe increases and the onset shifts to lower drive field, beginning at 25 Oe.

Related behavior has been observed in other nanowire samples in which field only[8] and electric current assisted[17] DW mobility curves were measured. The independent field only mobility measurements [Fig. 2 of Ref. [8]] reproduce the critical field peak at 5 Oe and the linearly increasing mobility behavior above 30 Oe shown in Fig. 8 but do not manifest the structure between 10 Oe and 30 Oe. The nanowire structure used in the electric current assisted mobility curve measurements essentially reproduced the $300 \mathrm{~K}$ mobility curve of Fig. 8, including the structure between 10 and 30 Oe drive field, but this structure vanished when high current density $j= \pm 5.8 \times 10^{11} \mathrm{~A} / \mathrm{m}^{2}$ was applied to the nanowire which also resulted in changes in the DW velocity at a prescribed drive field. These experiments suggest that 1) stable enhanced velocity modes are supported by Permalloy nanowires (as suggested by the simulations); 2) that these structures are sensitive to fabrication procedures, and 3) that they are not robust in the sense that applied high current density or elevated temperature can suppress the formation of the modes.

Comparison of the simulated [Fig. 10] and measured [Fig. 11] mobility curves reveals the absence of detailed agreement but some general correlations. While the enhanced velocity modes are not strikingly evident in the experiment, the simulations correctly predict the general features of the measured mobility curves including the two linear mobility regions, the approximate values of peak mobility and critical field, and the correct sign and approximate magnitudes of the temperature dependent shifts.

\section{EXPERIMENTAL EVIDENCE FOR THE SIMULATED DW BEHAVIOR}

All of the compact DW configurations described in Fig. 1 and the stretching mode identified in Fig. 5 have been observed by experiments capable of resolving spin distributions on the scale of a DW width. Some of the dynamical behavior described in relation to the simulated mobility curves (Fig. 5 and the associated spin distribution temporal evolution 
TABLE II. Selected references in which compact domain-wall types, illustrated in Fig. 1, are experimentally identified.

\begin{tabular}{ccccc}
\hline \hline & MFM & XMCD-PEEM & MTXM & Lorentz Microscopy \\
\hline TW(ATW) & Ref. [4] & Ref. [33] & & Ref. [35, 36] \\
VW & Ref. $[4,29,31]$ & Ref. [32, 33] & Ref. [34] & Ref. [35, 36] \\
DVW & Ref. [30, 31] & Ref. [32] & Ref. [34] & \\
TVW & & Ref. [32] & & \\
CT & Ref. $[4,30]$ & Ref. [32] & Ref. [34] & \\
\hline \hline
\end{tabular}

Figs. 7 and 8) have also been studied directly using high resolution spin sensitive microscopy, or indirectly using magneto optic time-of-flight and transient response techniques[10].

Table II lists selected references that describe observations of the compact DW spin distributions described in Fig. 1 (the results are limited to rectangular cross section Permalloy nanowires having dimensions corresponding to the phase diagrams of this study (Figs. 24). Various experimental techniques are represented including: magnetic force microscopy, Lorentz microscopy and photoelectron microscopy. One conclusion that can be drawn from Table II is that the simulations produce spin configurations and extended DW structures that are consistent with corresponding measurements of nanowires having widths of $100 \mathrm{~nm}$ or wider. Edge roughness associated with the most common nanowire fabrication techniques used in preparing the nanowire samples does not appear to play a dominant role in the formation of the static spin distributions. On a larger spatial scale, this result is analogous to the numerical simulations of the variety of closure domains that have been observed in thin film based rectangular microstructures. The stability of dynamic coupled vortex structures could be affected by edge effects associated with nanowire fabrication as discussed in the previous section.

\section{CONCLUSIONS}

This paper presents a systematic numerical simulation study of the static and dynamic spin distributions in rectangular cross-section Permalloy nanowires. Thin narrow nanowires support simple TW and VW structures, but more complex DW structures form as the 
nanowire width and thickness are increased. The stable compact DW types all share a topological property: the sum of their topological charge is zero. The local and global energies of the various DW configurations were used to construct a spin texture phase diagram that identifies phase boundaries and crossover boundaries for the various stable static spin distributions as a function of nanowire cross-section dimensions. Simulated crosssectional area dependent mobility curves exhibit systematic trends in drive-field dependent propagation modes that are related to the stable compact static spin distributions. Some of the complex structures including coupled vortex/antivortex structures are stable over fixed drive field ranges and propagate at significantly higher velocity than the modes at lower and higher drive fields. Images of DW structures verify the existence of all of the static compact DW structures identified by the numerical simulations, and experimentally determined mobility curves support the existence of the coupled voertex/antivorrex modes and the stretch mode that extends long distances along the nanowire. Simulations show that temperature variation around room temperature does not destroy the enhanced-velocity compact propagating modes, but does shift the drive-field ranges over which the modes are stable. Experimental measurements of mobility curves suggest that enhanced velocity modes are not robust and are affected by temperature and applied current. One final note: The numerical simulations of temperature effects in nanowire conduits presented in Section V do not account for all temperature-dependent magnetic phenomena in the nanowire structures studied in this report. Nanowire structures fabricated on Si (or other substrates) are subject to anisotropic stress resulting from different thermal expansion coefficients of the conduit $\left(\mathrm{Ni}_{80} \mathrm{Fe}_{20}\right)$ and the substrate structure $\left(\mathrm{SiO}_{2}\right.$ on $\mathrm{Si}$ with $\mathrm{Ta}$ buffer layers) in the specific case of the present study. The resulting uniaxial stress produces a magnetic anisotropy field $H_{\mathrm{K}}$ which affects spin precession resonance frequencies[37] and also can account for the apparent temperature-dependent shift in the mobility cures shown in Fig. 11. These effects are important, but are beyond the scope of the present work, but will be addressed in a future publication[38]. 


\section{ACKNOWLEDGMENTS}

This work was supported by the NSF DMR-1206404. Simulations were carried out using HPC resources of TACC at UT Austin.

[1] A. Hubert and R. Schäfer, Magnetic Domains (Springer, Berlin, 1998).

[2] D. A. Allwood, G. Xiong, M. D. Cooke, C. C. Faulkner, D. Atkinson, N. Vernier, and R. P. Cowburn, Science 296, 2003 (2002).

[3] D. A. Allwood, G. Xiong, C. C. Faulkner, D. Atkinson, D. Petit, and R. P. Cowburn, Science 309, 1688 (2005).

[4] S. S. P. Parkin, M. Hayashi, and L. Thomas, Science 320, 190 (2008).

[5] G. Vieira, T. Henighan, A. Chen, A. J. Hauser, F. Y. Yang, J. J. Chalmers, and R. Sooryakumar, Phys. Rev. Lett. 103, 128101 (2009).

[6] H. Lee, A. M. Purdon, and R. M. Westervelt, Appl. Phys. Lett. 85, 1063 (2004).

[7] P. Vavassori, M. Gobbi, M. Donolato, M. Cantoni, R. Bertacco, V. Metlushko, and B. Ilic, J. Appl. Phys. 107, 09B301 (2010).

[8] G. S. D. Beach, C. Nistor, C. Knutson, M. Tsoi, and J. L. Erskine, Nature Mater. 4, 741 (2005).

[9] G. S. D. Beach, C. Knutson, M. Tsoi, and J. L. Erskine, J. Magn. Magn. Mater. 310, 2038 (2008).

[10] J. Yang, C. Nistor, G. S. D. Beach, and J. L. Erskine, Phys. Rev. B 77, 014413 (2008).

[11] S. A. Yang, G. S. D. Beach, C. Knutson, D. Xiao, Q. Niu, M. Tsoi, and J. L. Erskine, Phys. Rev. Lett. 102, 067201 (2009).

[12] M. Tsoi, R. E. Fontana, and S. S. P. Parkin, Appl. Phys. Lett. 83, 2617 (2003).

[13] A. Yamaguchi, T. Ono, S. Nasu, K. Miyake, K. Mibu, T. Shinjo, Phys. Rev. Lett. 92, 077205 (2004).

[14] M. Yamanouchi, D. Chiba, F. Matsukura, and H. Ohno, Nature (London) 428, 539 (2004).

[15] M. Kläui, C. A. F. Vaz, J. A. C. Bland, W. Wernsdorfer, G. Faini, E. Cambril, L. J. Heyderman, F. Nolting, U. Rudiger, Phys. Rev. Lett. 94, 106601 (2005). 
[16] M. Kläui, P. -O. Jubert, R. Allenspach, A. Bischof, J. A. C. Bland, G. Faini, U. Rüdiger, C. A. F. Vaz, L. Vila, and C. Vouille, Phys. Rev. Lett. 95, 026601 (2005).

[17] G. S. D. Beach, C. Knutson, C. Nistor, M. Tsoi, and J. L. Erskine, Phys. Rev. Lett. 97, $057203(2006)$.

[18] R. D. McMichael and M. J. Donahue, IEEE Trans. Magn. 33, 4167 (1997).

[19] Y. Nakatani, A. Thiaville, and Miltat, J. Magn. Magn. Mater. 290-291, 750 (2005).

[20] S. Jamet, N. Rougemaille, J.C. Toussaint and O. Fruchart, 2014, arXiv:1412.0679.

[21] N. L. Schryer and L. R. Walker, J. Appl. Phys. 45, 5406 (1974).

[22] J.-Y. Lee, K.-S. Lee, S. Choi, K. Y. Guslienko, and S.-K. Kim, Phys. Rev. B 76, 184408 (2007).

[23] M. R. Scheinfein, LLG Micromagnetic Simulator ${ }^{T M}$, http://llgmicro.home.mindspring. com.

[24] Texas Advanced Computing Center (TACC), http://www.tacc.utexas.edu.

[25] N. W. Ashcroft and N. D. Mermin, Solid State Physics (Philadelphia, Saunders College, 1976).

[26] J. Fassbender, T. Strache, M. O. Liedke, D. Markó, S. Wintz, K. Lenz, A. Keller, S. Facsko, I. Möch, and J. McCord, New J. Phys. 11, 125002 (2009).

[27] U. Atxitia, D. Hinzke, O. Chubykalo-Fesenko, U. Nowak, H. Kachkachi, O. N. Mryasov, R. F. Evans, and R. W. Chantrell, Phys. Rev. B 82, 1134440 (2010).

[28] J. Yang, G. S. D. Beach, C. Knutson, and J. L. Erskine, J. Magn. Magn. Mater. 397, 325 (2016); J. Yang, G. S. D. Beach, C. Knutson, and J. L. Erskine, arXiv:1103.6056v1 (2011).

[29] S. Yang and J. L. Erskine, Phys. Rev. B 75, 220403(R) (2007).

[30] P. E. Roy, J. H. Lee, T. Trypiniotis, D. Anderson, G. A. C. Jones, D. Tse, and C. H. W. Barnes, Phys. Rev. B 79, 060407(R) (2009).

[31] M. H. Park, Y. K. Hong, B. C. Choi, M. J. Donahue, H. Han, and S. H. Gee, Phys. Rev. B 73, $094424(2006)$.

[32] M. Kläui, M. Laufenberg, L. Heyne, D. Backes, U. Rüdiger, C. A. F. Vaz, J. A. C. Bland, L. J. Heyderman, S. Cherifi, A. Locatelli, T. O. Mentes, and L. Aballe, Appl. Phys. Lett. 88, 232507 (2006).

[33] M. Laufenberg, D. Backes, W. Bührer, D. Bedau, M. Kläui, U. Rüdiger, C. A. F. Vaz, J. A. C. Bland, L. J. Heyderman, F. Nolting, S. Cherifi, A. Locatelli, R. Belkhou, S. Heun, and E. Bauer, Appl. Phys. Lett. 88, 052507 (2006). 
[34] G. Meier, M. Bolte, R. Eiselt, B. Krüger, D.-H. Kim, and P. Fischer, Phys. Rev. Lett. 98, $187202(2007)$.

[35] M. Eltschka, M. Wötzel, J. Rhensius, S. Krzyk, U. Nowak, M. Kläui, T. Kasama, R. E. DuninBorkowski, L. J. Heyderman, H. J. van Driel, and R. A. Duine, Phys. Rev. Lett. 105, 056601 (2010).

[36] Y. Togawa, T. Kimura, K. Harada, T. Akashi, T. Matsuda, A. Tonomura, and Y. Otani, Jpn. J. Appl. Phys. 45, L683 (2006).

[37] K. An, X. Ma, C.-F. Pai, J. Yang, K. S. Olsson, J. L. Erskine, D. C. Ralph, R. A. Buhrman, and X. Li, Phys. Rev. B 93, 140404(R) (2016).

[38] J. Yang, K. Mun, K. An, X. Li, and J. L. Erskine (unpublished). 